\title{
Estudio diagnóstico sobre hábitos relacionados con estilos de vida saludable en alumnos de una escuela primaria
}

\begin{abstract}
A Diagnostic Study About Healthy Lifestyle Habits in Elementary School
Students
\end{abstract}

Estudo diagnóstico sobre hábitos relacionados ao estilo de vida saudável em escolares do ensino fundamental

Denisse Alejandra Unzueta Vargas

Universidad Juárez del Estado de Durango, México denisse.uv@gmail.com https://orcid.org/0000-0001-5010-6266

Jaime Fernández Escárzaga*

Universidad Juárez del Estado de Durango, México jaimefer14@hotmail.com https://orcid.org/0000-0002-8897-5851

Patricia Lorena Martínez Martínez Universidad Juárez del Estado de Durango, México patitomar@hotmail.com https://orcid.org/0000-0003-4860-1625

*Autor de correspondencia 


\section{Resumen}

La promoción de estilos de vida saludable ha cobrado importancia en los últimos años debido a los altos índices de sobrepeso y obesidad que presentan los niños en edad escolar en México. Sin embargo, los resultados obtenidos después de más de 10 años de implementar diferentes estrategias indican que los esfuerzos no han generado el impacto esperado. Por esto, la atención se ha colocado en las escuelas, en tanto que estas deberían de ser comunidades de promoción social: espacios donde se adopten modelos inclusivos que promuevan acciones e implementen políticas a fin de proporcionar a los alumnos herramientas y recursos personales para superar las barreras que limitan el desarrollo integral, como pueden ser las condiciones de salud, higiene y hábitos poco saludables influenciados por el contexto sociocultural.

El objetivo de este estudio fue elaborar un diagnóstico que revelara cuáles son las prácticas inclusivas que se realizan en un entorno escolar en relación con los estilos de vida saludable. Se llevó a cabo en una escuela de educación primaria de la periferia de la ciudad de Durango, Durango, México; y se utilizó un enfoque cualitativo de investigación de tipo observacional fenomenológico. Los resultados encontrados indican que los alumnos están en situación de exclusión y vulnerabilidad debido a las condiciones socioculturales de precariedad que tienen que enfrentar, por lo que resulta importante dar atención a la necesidad de mejorar las condiciones de salud, higiene y hábitos de estilo de vida saludable de los alumnos para que se den las condiciones para lograr conseguir un bienestar capaz de conducir al desarrollo de su potencial humano. El diagnóstico que aquí se presenta generó los elementos necesarios para desarrollar un proceso de intervención fundamentado en la psicología educativa con un modelo de investigación-acción, del cual aún no se pueden reportar resultados debido a que está todavía en período de intervención.

Palabras clave: calidad de vida, estilo de vida, psicología educacional. 


\section{Revista Iberoamericana \\ de las Ciencias Sociales y Humanísticas}

ISSN: $2395-7972$

\section{Abstract}

Healthy lifestyle promotion has been an important issue during the last years in response of the high rates of overweight and obesity in the children population in our country. However, after more than 10 years of implementing different strategies against this health problems, the results show that effects are quite poor. This is the main reason why schools have become the central focus in the promotion of healthy lifestyles, because experts found that schools should be transformed into communities for social promotion: spaces where inclusive education models that gives inner resources to their students by promoting actions and implementing policies that eliminate all the obstacles in the full development of children are adopted, such as health and hygiene conditions or unhealthy lifestyle habits because of the sociocultural context.

The objective of the study was to make a diagnosis that reveals the inclusive practices that are carried out in a school environment in terms of healthy lifestyle. It was made in a public elementary school located in the outskirts of the city of Durango, Durango, México. The method used in the diagnosis was qualitative with a phenomenological observational type. The results show that students are socially excluded and vulnerable as a result of the poverty conditions they have to deal, and that's the reason why is important to improve the lifestyle habits, hygiene and health conditions in order to get close to their well-being and can get a better development of their human potential. This diagnosis provided enough elements for develop an intervention process with an action-research orientation guided by educational psychology bases, which is still in phase of implementation in the school community.

Keywords: quality of life, lifestyle, educational psychology.

\section{Resumo}

A promoção de estilos de vida saudáveis ganhou importância nos últimos anos devido às altas taxas de sobrepeso e obesidade apresentadas pelas crianças em idade escolar no México. No entanto, os resultados obtidos após mais de 10 anos de implementação de diferentes estratégias indicam que os esforços não geraram o impacto esperado. Portanto, tem sido dada atenção às escolas, embora devam ser comunidades de promoção social: espaços onde modelos inclusivos são adotados, que promovem ações e implementam políticas, a fim de 


\section{Revista Iberoamericana}

de las Ciencias Sociales y Humanísticas

ISSN: $2395-7972$

fornecer aos alunos ferramentas e recursos pessoais para superar barreiras que limitam o desenvolvimento integral, como condições de saúde, higiene e hábitos não saudáveis influenciados pelo contexto sociocultural.

O objetivo deste estudo foi desenvolver um diagnóstico que revelasse quais são as práticas inclusivas realizadas no ambiente escolar em relação a estilos de vida saudáveis. Foi realizado em uma escola primária na periferia da cidade de Durango, Durango, México; e foi utilizada uma abordagem qualitativa de pesquisa do tipo observacional fenomenológico. Os resultados encontrados indicam que os estudantes estão em situação de exclusão e vulnerabilidade devido às condições socioculturais de precariedade que precisam enfrentar, por isso é importante prestar atenção à necessidade de melhorar os hábitos de saúde, higiene e estilo de vida. saudável para os alunos atenderem às condições para alcançar um bem-estar capaz de levar ao desenvolvimento de seu potencial humano. $\mathrm{O}$ diagnóstico aqui apresentado gerou os elementos necessários para o desenvolvimento de um processo de intervenção baseado na psicologia educacional, com um modelo de pesquisa-ação, cujos resultados ainda não podem ser relatados porque ainda estão no período de intervenção.

Palavras-chave: qualidade de vida, estilo de vida, psicologia educacional.

Fecha Recepción: Enero 2019

Fecha Aceptación: Junio 2019

\section{Introducción}

En este artículo se presentan los resultados del diagnóstico realizado en una escuela primaria ubicada en la periferia de la ciudad de Durango, Dgo., México. Se evaluaron las prácticas relacionadas con estilos de vida saludable. Dichos estilos son considerados un indicador de educación inclusiva.

En el año 2010, la Secretaría de Educación Pública (SEP) sostuvo el Acuerdo Nacional para la Salud Alimentaria como una estrategia para combatir los altos índices de obesidad y sobrepeso en los niños. Allí se planteaban acciones para la promoción y educación para la salud; sin embargo, se dejó de aplicar porque resultó ser insuficiente para generar una cultura escolar favorecedora de estilos de vida saludable.

El Acuerdo del 2010 no se llevó a cabo de forma eficiente o no era lo adecuado para la magnitud del problema. La situación de salud alimentaria en los alumnos de educación primaria persiste. De ahí que es importante continuar estudiando cómo promover los estilos 


\section{Revista Iberoamericana}

de las Ciencias Sociales y Humanísticas

ISSN: 2395 - 7972

de vida saludable en los niños, por lo que se realizó un diagnóstico para analizar la situación escolar y sus condiciones socioeconómicas y culturales que determinan las prácticas de alumnos y docentes en la vida cotidiana de la escuela. La perspectiva teórica desde la que se observó el problema es desde la inclusión educativa.

La elaboración del diagnóstico posibilitó el diseño de una propuesta de intervención que, tomando como base las necesidades detectadas en el centro escolar, ofreciera a la institución la posibilidad, en primer lugar, de ser conscientes de sus prácticas de inclusión, y con ello, en un segundo momento, establecer las condiciones para potenciarse como un espacio estimulante con el fin de desarrollar sujetos sanos e integrados; todo ello para cumplir sus objetivos más esenciales: educar y formar (Banz, 2004, p. 4).

Como antes ya se dijo, para alcanzar el objetivo de transformar las prácticas y políticas del centro escolar y orientarlas hacia la adopción de estilos de vida saludable, se utilizó el enfoque de inclusión educativa, debido a que esta filosofía de trabajo trata de la participación de todos los estudiantes, docentes y padres de familia a través del establecimiento de alianzas como parte de un proceso de mejora educativa, interviniendo en áreas como la promoción de la salud (Booth y Ainscow, 2015, p. 16). Es por eso por lo que se adopta el enfoque de inclusión educativa desde el momento de la realización del diagnóstico.

\section{Antecedentes}

Según datos de la Encuesta Nacional de Salud y Nutrición 2012 (Ensanut), $34.4 \%$ de los niños mexicanos entre 5 y 11 años de edad presentan una prevalencia combinada de sobrepeso y obesidad; esto es, uno de cada tres niños tiene problemas de nutrición (Instituto Nacional de Salud Pública [INSP], 2012, p. 28). Esta misma encuesta señala que este porcentaje no muestra variación significativa en el periodo 2006-2012.

La tendencia continúa sin muchos cambios $\mathrm{y}$, desafortunadamente, sin muchas acciones que, durante 10 años, se hayan emprendido con éxito. En la Ensanut realizada en 2016, la prevalencia de sobrepeso y obesidad para niños en edad escolar (5 a 11 años) fue de $33.2 \%$; sin embargo, se especifica que los intervalos de confianza de la prevalencia de 2016 son relativamente amplios, por lo que no es posible concluir que la prevalencia combinada de sobrepeso y obesidad disminuyó en comparación con los resultados del 2012 (INSP, 2016, p. 64). 


\section{Revista Iberoamericana de las Ciencias Sociales y Humanísticas}

ISSN: $2395-7972$

Debido a estas estadísticas, en las que se muestra una tendencia de estabilidad en los porcentajes de sobrepeso y obesidad infantil, durante los últimos años en México se han estado realizando diversas campañas y programas orientados a la promoción de un estilo de vida saludable, pero la mayoría quedan en campañas de información.

La SEP, en coordinación con la Secretaría de Salud, pusieron en operación el ya mencionado Acuerdo Nacional para Salud Alimentaria: Estrategia contra el Sobrepeso y Obesidad, el cual contaba con los componentes de promoción y educación para la salud, el fomento de la actividad física regular, así como acceso y disponibilidad de alimentos y bebidas que faciliten una alimentación correcta, regulando la venta de alimentos "chatarra" dentro de las instituciones educativas (SEP, 2010, p. 11). Pero son programas paralelos al diseño curricular, que no se implementan en todas las localidades y resultan ser de aplicación temporal y de resultados magros.

En el año 2012, con el cambio de gobierno, se impusieron nuevas políticas educativas y la implementación de dicho acuerdo cesó. Actualmente, en el Modelo Educativo para la Educación Obligatoria, la promoción hacia un estilo de vida activo y saludable se incluye en el ámbito "Atención al cuerpo y la salud" (SEP, 2017, p. 51), integrando los conocimientos de esta área como parte del componente curricular en las asignaturas de Ciencias Naturales y Educación Física. Habrá que esperar a ver si llega la capacitación a los docentes para la ejecución de este nuevo plan tendiente a mejorar los hábitos de salud, el cual deberá ser aplicado no solamente en los alumnos, sino dentro la comunidad en donde se encuentra inserta la escuela.

Existen estudios en los que se reconoce la influencia de los centros escolares en la promoción de hábitos de estilo de vida saludable. Tal es el caso del estudio descriptivo realizado por Campo, Herazo, García, Suárez, Méndez y Vásquez (2017) en el que elaboraron una caracterización diagnóstica como parte del proyecto de Promoción de Estilos de Vida Saludables en niños mayores de 6 años y adolescentes de 16 escuelas públicas en Barranquilla, Colombia.

Para la elaboración del estudio se analizaron los niveles de actividad física en el contexto escolar, así como hábitos alimenticios, de higiene corporal, bucal y lavado de manos. La conclusión a la que arribaron fue de que es fundamental enfocar diversas estrategias de educación en salud; además de ampliar el espectro investigativo a la búsqueda 


\section{Revista Iberoamericana de las Ciencias Sociales y Humanísticas}

ISSN: $2395-7972$

de relaciones entre variables y factores determinantes de los estilos de vida en este grupo poblacional (Campo et al., 2017, pp. 425-426).

En México también se han realizado investigaciones en las que se destaca la necesidad de modificar el estilo de vida a través de intervenciones en los centros escolares. Un ejemplo de ello es el estudio realizado por Arvide, Rodríguez, Henao, Gómez y Aguilar (2013), quienes aplicaron un programa de adopción de estilo de vida saludable por un período de tres años en escuelas primarias del Estado de México. La intervención consistió en proporcionar educación sobre hábitos saludables, modificación de los alimentos distribuidos y actividad física. En este caso se obtuvo como resultado un menor incremento en el índice de masa corporal y como conclusión, que los programas escolares son útiles para enfrentar la obesidad infantil, aun cuando sus beneficios no son inmediatos.

\section{Consideraciones teóricas}

En sus concepciones más actuales, el estilo de vida se concibe como un conjunto de decisiones que las personas toman en día a día y que afectan su salud. Los individuos tienen más o menos control sobre tales decisiones, de modo que el estilo de vida saludable se comprende como el conjunto de estos patrones de conducta relacionados con su salud. La relevancia del estudio de la vida saludable se determina por el hecho de que las personas tienen la condición de realizar elecciones, en el marco de opciones disponibles o en el contexto de oportunidades que ofrece la propia vida (Cockerham, 2007, p. 327).

De acuerdo con lo anterior, los determinantes del estilo de vida relacionados con la salud son diversos, y su estudio puede abordarse desde dos perspectivas distintas: una que se interesa en la dinámica del sujeto, tales como en su individualidad, que refleja unidad de estilo y de acción en la conducta de las personas; y otra que transita en la interrelación sujetocultura y refiere el término tanto desde aspectos contextuales como de aprendizaje colectivo (Cokerham, Rütten y Abel, 1997; citados en Muñoz y Uribe, 2013, p. 357).

En este contexto, algunos estudios valoran aspectos individuales, como son los factores biológicos y la conducta relacionada con comportamientos asociados con enfermedad. Otros, en cambio, buscan analizar elementos comportamentales inmersos en lo sociocultural, a saber: la actividad física, los hábitos alimenticios, el consumo de alcohol, tabaco y otras drogas, la recreación y el manejo del tiempo libre, el autocuidado, el cuidado médico y el sueño (Arrivillaga, Salazar y Correa, 2003, p. 186). En consecuencia, es clara la 


\section{Revista Iberoamericana \\ de las Ciencias Sociales y Humanísticas}

ISSN: 2395 - 7972

relevancia de las variables sociológicas y socioeconómicas en el tema, tales como educación, estrato social y pobreza. Lo anterior sin dejar de lado las particularidades del ser humano que obedecen a su historia personal.

El estilo de vida se va formando a lo largo de la existencia de las personas, por medio de un proceso de aprendizaje, en el cual se imitan patrones familiares y sociales que conducen a adquirir conductas propias del ambiente. No obstante, el estilo de vida no se forma únicamente de manera espontánea, sino que es posible que las personas tomen la decisión de modificarlo para intentar ser mejores y mejorar la sociedad de la que son parte (Sanabria, 2007, p. 209).

Uno de los factores contextuales que se ha relacionado con este tópico y que poco se ha estudiado es el estrato socioeconómico. Estudios realizados en Latinoamérica reportan que esta variable se asocia al estilo de vida; se afirma la existencia de una relación directamente proporcional entre ambos aspectos. Muñoz y Uribe (2013, p. 359), por ejemplo, concluyen que a mayor estrato, mayor presencia de creencias favorables de salud.

Cuando las formas de vivir y las pautas de conductas habituales de las personas y grupos contribuyen a la promoción y protección de la salud integral, se habla de estilos de vida saludables; es decir, un estilo de vida saludable denota comportamientos que disminuyen los riesgos de enfermar, tales como un adecuado control y tratamiento de las tensiones y emociones negativas, un buen régimen deportivo, sueño, diversiones y una correcta distribución del tiempo, entre otros (Vives, 2007; citado en Grimaldo, 2012, pp. 75-76).

Al hablar de estilos de vida saludable, es reciente el interés por estudiar el tema en niños y adolescente. González, Gómez, Valtueña y Meléndez (2008) advirtieron de la necesidad de elaborar un instrumento educativo desarrollado específicamente para estos grupos de edad.

Consecuentemente, la pirámide del estilo de vida saludable planteada por González et al. (2008) resulta innovadora puesto que incluye como puntos fundamentales la atención a los hábitos de higiene y cuidados de salud que los niños y adolescentes deben adoptar durante toda la vida, tales como lavarse los dientes después de cada comida, bañarse diariamente, lavarse las manos de manera periódica, masticar bien los alimentos, acudir con regularidad a revisiones médicas y odontológicas, así como evitar el consumo de sustancias dañinas como el alcohol, tabaco o drogas (González et al., 2008). Y es en este ámbito en el 


\section{Revista Iberoamericana \\ de las Ciencias Sociales y Humanísticas}

ISSN: 2395 - 7972

cual los centros escolares pueden ser un factor de reforzamiento importante en la promoción de hábitos relacionados con un estilo de vida saludable en los estudiantes.

En relación con el desarrollo integral de los estudiantes, la Encuesta Nacional de Salud en Escolares 2008 (INSP, 2010) destaca que el rendimiento escolar está relacionado con diversos factores: los entornos familiar, social, ambiental y socioeconómico; además de la carencia de servicios médicos para prevenir y atender enfermedades. Y deja en claro que los problemas de salud asociados con la alimentación y la nutrición afectan la capacidad de aprendizaje de manera muy significativa.

Por este motivo, es que se considera que los centros educativos pueden convertirse en el medio ideal para que las personas realicen un análisis crítico de los problemas de salud que se presentan en la comunidad educativa y proporcionar, mediante un plan de mejora, acciones específicas que ayuden a los individuos a desarrollar las herramientas necesarias para responsabilizarse de su situación y tomar decisiones orientadas a mejorar su salud y generar hábitos de vida saludable.

Ningún acuerdo intersecretarial, ley o modelo educativo va a prosperar si no se atiende desde la raíz el problema de la institución educativa. A lo largo de la historia se han observado muchísimos programas emergentes que solamente quedan en buenas intenciones. Situación que no solamente sucede con lo extracurricular, sino con el curriculum formal, y acontece de esa manera porque la institución escolar no asume que es su responsabilidad todo lo que ocurre en su interior.

Los niños que acuden a la escuela pública en zonas marginales, como es el caso del centro estudiado aquí, son alumnos que tienen muchas privaciones de tipo social, económico y cultural. En su familia, en su barrio, o en su círculo más próximo; difícilmente podrán encontrar un referente que les conduzca a llevar una vida más saludable y con mejores hábitos alimenticios. Si ese referente no proviene de la escuela, el niño tiene escasas probabilidades de obtenerlo de otra parte. Y si, como la mayoría de las veces ocurre, la escuela tan solo se dedica a reproducir los patrones culturales que pertenecen a la misma comunidad del alumno, no habrá transformación de hábitos para este último.

La inclusión educativa abarca dos grandes dimensiones: la de construir culturas inclusivas y la de establecer políticas inclusivas (Booth y Ainscow, 2015), en donde, desde luego, se encuentran los hábitos de vida saludable, de solidaridad, de conocimiento sobre los estilos de vida de la comunidad, etc. La formación integral planteada en esta filosofía 


\section{Revista Iberoamericana}

de las Ciencias Sociales y Humanísticas

ISSN: 2395 - 7972

educativa es el centro del trabajo docente, pero, como se afirma líneas arriba, es responsabilidad de la institución escolar que ocurra; son lineamientos que ya están establecidos, solo falta que se hagan realidad en cada centro escolar. La vida saludable de las personas que padecen un alto nivel de marginalidad solo podrá llevarse a cabo ciertamente en la escolaridad, que no debe reproducir irracionalmente pautas de vida cultural vigentes, sino que ha de comenzar a transformar a la sociedad a través de la formación escolarizada.

\section{Metodología}

Se realizó un estudio cualitativo observacional de tipo fenomenológico. Durante seis semanas se hizo observación no participante y se registraron en la bitácora y diario de campo todos los hechos que tuvieran relación con el estilo de vida de los miembros de la comunidad escolar, así como las prácticas docentes y políticas que favorecieran o dificultaran la inclusión al interior de la institución. La información se recopiló a lo largo de 16 sesiones realizadas en el horario de jornada escolar en una escuela primaria que presenta características de una comunidad rural, aun y cuando se encuentra ubicada en la periferia de la ciudad de Durango, Dgo., México.

Además, se hicieron entrevistas a profundidad a informantes clave: dos docentes y el director; un muestreo por conveniencia se realizó, debido a que estas figuras, a lo largo de las sesiones de observación, demostraron tener un papel de liderazgo o relevancia en la dinámica al interior de la institución educativa. Previo a la entrevista se comunicó a los informantes clave sobre el uso que se haría de la información solicitada, refiriendo que se manejarían con confidencialidad todos los datos; una vez obtenido el consentimiento explícito para participar en la investigación se procedió a la entrevista. Las entrevistas tuvieron una duración aproximada de una hora, en la que se indagó sobre las prácticas y políticas implementadas por la escuela para la promoción de salud, alimentación adecuada y cuidado personal en los alumnos; barreras culturales que obstaculizan las prácticas docentes, formas de participación de los padres de familia, valores que se fomentan en los alumnos, así como la dinámica de convivencia entre padres de familia, docentes y alumnos.

Posterior a la recopilación de información, esta fue organizada en cinco categorías: 1) Estilo de vida, 2) Manejo de la disciplina, 3) Políticas institucionales, 4) Estilo de liderazgo en directivos y 5) Formas de participación de padres de familia. 
Para categorizar la información se analizó el discurso de los sujetos entrevistados; para construir las categorías, las notas de la observación, y se empleó el programa Atlas.ti para facilitar su agrupación. Para referenciar la transcripción de las notas de campo, los registros de las observaciones y de las entrevistas que se presentan en la continuación se recurrió a la siguiente simbología: entre paréntesis se indica con una $p$ a la persona y posteriormente se indica el número que se le asignó a fin de diferenciar a los interlocutores, y después la posición en donde se encuentra en la bitácora el texto en referencia.

El proceso de análisis de la información produjo cinco categorías, de las cuales en este artículo solamente se presentan las que se encuentran relacionadas con el estilo de vida, debido a que utilizar todas las categorías implica una extensión muy amplia del reporte. La definición que se construyó para esas categorías se presenta en la tabla 1. 
Revista Iberoamericana

de las Ciencias Sociales y

Humanísticas

ISSN: $2395-7972$

Tabla 1. Definición de las categorías encontradas en el diagnóstico

\begin{tabular}{|l|l|l|}
\hline \multicolumn{2}{|c|}{ Categoría: Estilo de vida } \\
\hline $\begin{array}{l}\text { Contexto social de la comunidad } \\
\text { escolar }\end{array}$ & $\begin{array}{l}\text { Comprende la descripción de las condiciones } \\
\text { sociales, económicas y culturales de las familias } \\
\text { bajo las cuales se han desarrollado los niños, y } \\
\text { que ejercen influencia en las expectativas que } \\
\text { los padres y maestros tienen sobre el futuro de } \\
\text { los niños, así como en el grado de participación } \\
\text { e involucramiento en las actividades escolares } \\
\text { relacionadas con el proceso de aprendizaje. }\end{array}$ \\
\hline 2) Hábitos de alimentación & $\begin{array}{l}\text { Hace referencia a todas las prácticas } \\
\text { relacionadas con el tipo de alimentación que los } \\
\text { niños tienen en sus hogares y en la escuela, las } \\
\text { cuales impactan de manera positiva o negativa } \\
\text { en el desempeño de los alumnos. }\end{array}$ \\
\hline 3) Hábitos de higiene de los alumnos & $\begin{array}{l}\text { Abarca todas las prácticas y conductas que los } \\
\text { alumnos realizan como parte de su vida } \\
\text { cotidiana que son indicadores de la limpieza, } \\
\text { aseo y cuidado que los niños tienen sobre sí } \\
\text { mismos, así como de sus entornos más } \\
\text { próximos, como lo son la escuela y sus casas, } \\
\text { que influyen en el estado de salud de los } \\
\text { alumnos y el estilo de vida que llevan. }\end{array}$ \\
\hline 4) Condiciones de higiene escolar & $\begin{array}{l}\text { Se refiere a todas aquellas prácticas que se } \\
\text { llevan a cabo dentro de la escuela, tanto por } \\
\text { alumnos y padres de familia como por el } \\
\text { personal de la institución, y que impactan en la } \\
\text { limpieza y sanidad de las áreas en las que los } \\
\text { alumnos realizan sus actividades de manera } \\
\text { cotidiana, ya sea implicando un adecuado } \\
\text { manejo de la higiene o constituyendo focos de } \\
\text { atención para la mejora del desarrollo de los } \\
\text { alumnos. }\end{array}$ \\
\hline
\end{tabular}

Fuente. Elaboración propia

\section{Resultados}

\section{Categoría 1: Contexto social de la comunidad escolar}

El contexto social en el cual han sido educados los alumnos de la escuela primaria ubicada en la periferia del norte de la ciudad de Durango, México, presenta algunas características importantes a considerar: es una comunidad periférica que funciona como zona rural, los servicios son limitados, no hay calles pavimentadas, y algunos de sus habitantes son trabajadores del campo, otros laboran en una fábrica aledaña y algunos más en la construcción en la ciudad. Las casas habitación son similares a las de la zona rural en 


\section{Revista Iberoamericana \\ de las Ciencias Sociales y Humanísticas}

ISSN: 2395 - 7972

su tipo de construcción, por lo que generalmente cuentan con una sección de corral, en donde tienen algunos animales como vacas, caballos o cerdos.

La mayoría de los alumnos tienen papás jóvenes con un rango de edad entre los 20 y 35 años, quienes abandonaron sus estudios y laboralmente se desempeñan como obreros. Al respecto, informó un docente: “Trabajan recogiendo basura, otros en minas, unos más solo saben que sus papás viven lejos pero no saben en qué trabajan" (p3,128:128). Los padres de familia tienen que salir a trabajar para asegurar el sustento de su familia, por lo que dedican poco tiempo al cuidado y convivencia con sus hijos; tampoco lo hacen en la supervisión o en la participación de actividades escolares por la poca preparación académica que en algunos casos llegan a tener: "Los papás no se involucran, no tienen compromiso para hacerlo, pero tampoco la preparación. No les explican o ayudan en las tareas, se las hacen para no batallar" (p3, 360:360). Esa es la percepción de los docentes de la escuela primaria, y como generalmente sucede, la descarga de la responsabilidad es hacia los demás: siempre hacia fuera de la institución escolar, casi nunca hacia dentro. En la escuela prácticamente nadie se pregunta: ¿Qué debemos hacer para solucionar este problema? ¿Podríamos implementar un programa para enseñar a los padres a educar a sus hijos, a que aprendan cómo apoyar nuestra labor docente?

En lo referente a la composición sociocultural arraigada en el contexto de los alumnos, se encontró que el consumo de alcohol, tabaco y drogas es una práctica común para los niños, quienes lo viven como parte de su día a día y lo ven como algo natural: están expuestos al consumo temprano de estas sustancias:

Veo cinco niñas trabajando en anuncios para venta de cerveza Corona o Tecate Light. Estoy tomando notas cuando un niño se me acerca y me dice: "Maestra, ¿para qué sirven los remedios de la marihuana? Para las reumas, y ¿para qué más?". Se va a seguir trabajando en su anuncio y veo que su trabajo se titula "Venta de mota El Jonny" (entrevistador 1, 93:93).

Estas características del contexto social de la comunidad escolar, en gran parte determinadas por el nivel socioeconómico tendiente a la precariedad, condicionan el acceso de los alumnos a un desarrollo integral, pues influyen en aspectos tales como los siguientes: 


\section{Revista Iberoamericana \\ de las Ciencias Sociales y Humanísticas}

ISSN: $2395-7972$

- Las expectativas de los padres de familia sobre el papel de la escuela en la educación de sus hijos, pues conciben a la escuela "como guardería (...). [Van y dejan] a sus hijos, se van a trabajar a Macisa [fábrica aledaña] por 600 pesos a la semana, no tienen aspiraciones, y pues los niños tampoco" (p3, 217:217).

- La posibilidad de adquirir los materiales necesarios para las actividades académicas de los niños. Situación que reporta un maestro cuando les pide a sus alumnos que asistan con cierta vestimenta: "Los niños con camisa negra, de cuello alto de preferencia (...), aunque si no hay pa más, con lo que tengan” (p3, 111:111).

- Las condiciones de higiene y cuidado que los alumnos tienen de sí mismos: "He tratado de mejorar a los niños en términos de aseo y limpieza, pero se quejan que soy muy estricto, y nuevamente recibo poco apoyo de los papás” (p3, 176:176).

- Las expectativas que los maestros tienen acerca de las capacidades académicas de sus alumnos. Debido a que se detectó en la práctica docente poca expectativa de cambio respecto a las condiciones de vida en las que actualmente se desempeñan sus alumnos. Obsérvese en el siguiente diálogo cómo la tendencia a que los niños tengan pocas aspiraciones es claramente notoria:

Les digo: "A ver, cuando trabajen en Teleperformance o en Soriana, van a trabajar con computadoras y ahí les van a pedir que estén concentrados y en calma”. Les pongo esos ejemplos porque son los lugares en los que pueden llegar a trabajar, entonces que vayan sabiendo de una vez (p3, 97:97).

\section{Categoría 2: Hábitos de alimentación}

Respecto a la categoría Hábitos de alimentación, se observó como práctica común el que las madres de familia asisten diariamente a llevar desayunos a sus hijos a la hora del recreo, y se detectó igualmente que los alimentos consumidos por los alumnos son de alto contenido calórico y poco contenido nutricional, y en la escuela prácticamente no se hace nada al respecto. Cabe señalar que la alimentación de los docentes en ese mismo sitio no es muy diferente a la de los alumnos. Uno de los informantes clave entrevistado dice a este respecto: 
Revista Iberoamericana

de las Ciencias Sociales y

Humanísticas

ISSN: 2395 - 7972

Entre los alimentos que consumen [los alumnos] se pueden observar tamales, tortas, burritos, conchas de chocolate, churritos con salsa, refrescos, jugos envasados de diferentes sabores. Muchos niños, después de comer su refrigerio, van y compran dulces en el puestito: bombones, paletas, chilitos en polvo, chicles Totito, cachetadas, etc. (p3, 11:11).

Algunos docentes son conscientes de la necesidad de modificar estos hábitos de alimentación en los alumnos. Por ello han hecho intentos de incluir a las madres en la enseñanza de conductas que favorezcan cambios en los hábitos de alimentación. Esta idea de crear alianzas entre la escuela y los padres de familia para la modificación en los hábitos de alimentación de los niños ha sido impulsada por algunos docentes, a decir del director.

Algunos maestros implementaron una dinámica en la cual les hacían traer a los muchachos ingredientes y ellos tenían que hacer porciones en un plato acerca de cómo debería de ser el plato del buen comer (...). Las madres de familia también participan trayendo alimentos que están dentro del plato y se hace una comidita sencilla (...). La intención es que los niños vengan y participen de los mismos conocimientos a las madres de familia, y que ellos mismos exijan una alimentación sana y como debe corresponder (p6, 13:13).

Sin embargo, estos intentos no han generado el impacto que se esperaba. Al respecto, dice una maestra:

Lo que pasa es que en muchas ocasiones el gran impedimento es la cuestión económica, porque, por ejemplo, los niños dicen "no debo comer esto o debo comer esto otro, pero en mi casa no lo comemos o por falta de hábitos o por cuestiones económicas" (p8, 14:14).

Por una parte, es la cultura y la determinante económica de las familias, pero, por otro lado, se hace presente la actuación de la escuela, en donde al parecer si algo es difícil y conlleva mucho trabajo y a largo plazo, no se hace. Transformar hábitos nunca es sencillo, hay experiencias de éxito en muchas comunidades, pero desafortunadamente son eventos aislados. 


\section{Categoría 3: Hábitos de higiene de los alumnos}

Continuando con el análisis de estilo de vida, en la categoría Hábitos de higiene de los alumnos" se encontró que el baño diario es un hábito que no han adquirido muchos de los niños, situación que se hace patente de diversas maneras. Uno de los informantes lo pone en los siguientes términos: "Si yo me quedo ahí [en el salón], porque no tengo opción, y si ahorita huele feo, no le quiero contar cómo huele en mayo. Pero estos niños no entienden, uno les dice que se bañen y no hacen caso" (p3, 198:198). El hábito de lavarse los dientes después de cada comida es igual de importante que el del baño diario; sin embargo, este hábito tampoco es frecuente en los niños, motivo por el cual los maestros llevan a cabo acciones para promover la salud bucal en sus estudiantes. Uno de ellos está muy pendiente de esto con sus alumnos, pero solamente es uno; él les dice después de que consumen alimentos: "Agarren sus cepillos de dientes todos y vayan a lavárselos, y ya saben, para entrar tienen que soplarme y debe oler a limpio, si no no entran" (p3, 340:340).

Otro aspecto importante en lo que se refiere a las condiciones de higiene de los alumnos es la presencia de piojos en sus cabezas (pediculosis capitis). Varios de los maestros entrevistados reportaron esto como un problema frecuente. A decir de una maestra: "Hay que revisarles a los niños los piojos que no traigan piojos, porque ese es un grave problema también aquí” (p6, 35:35). En la escuela solamente se toman acciones correctivas cuando el problema es fuerte; de lo contrario, se hace el intento de decirles a los niños que se bañen a diario, pero no se emprenden estrategias para transformar ese hábito.

\section{Categoría 4: Condiciones de higiene escolar}

Por último, en la categoría Condiciones de higiene escolar"no solamente son considerados los hábitos personales, sino que también son parte de ella lo que ocurre en el contexto del propio centro escolar. En el caso de la escuela que se analiza, una problemática observada es la presencia habitual de perros dentro de la escuela, principalmente a la hora del recreo, los cuales regularmente permanecen en las áreas comunes en las que tanto alumnos como maestros ingieren sus alimentos, a la espera de un descuido de alguno de los niños para obtener un poco de comida.

Uno de los riesgos de que los niños convivan con los perros al interior de la escuela es el hecho de que los animales están evidentemente descuidados y sucios, por lo que 
Revista Iberoamericana

de las Ciencias Sociales y

Humanísticas

ISSN: $2395-7972$

representan posibles focos de infección. La situación es descrita por una maestra de la siguiente manera:

Los niños aquí están acostumbrados a tirarse al piso, a revolcarse en el pasto y demás. ¿Con qué higiene y seguridad podemos nosotros decirles a los niños "sí, tírense al piso" cuando en el piso hay desde garrapatas, puede haber pulgas, piojos, como ya lo mencioné? ¡No! (p6, 49:49).

El personal docente y directivo reconoce estos riesgos para la salud de los niños, motivo por el cual han llevado a cabo acciones con la intención de sacar de la institución a los perros. "Se le ha hablado a la perrera municipal"; y también:

Tenemos una tela gallinera, que esa si la observa usted, pues cualquier perrito llega y con las patitas la levantan; necesitaríamos una tela borreguera de alambre grueso, bien soldadita, bien amarrada, para tapar el paso a los animales, y ya nada más controlar la puerta de entrada (p7, 42:42).

Estas acciones han sido infructuosas, pues los perros son mascotas de los alumnos, y en muchas ocasiones los mismos padres de familia llevan a los perros a la escuela, especialmente las madres de familia que todos los días van a llevar de desayunar a sus hijos a la hora del recreo. En la escuela se han realizado juntas con padres de familia en las que se les solicita que sean responsables y que no lleven a sus mascotas, pues representan un riesgo para la seguridad de los niños, debido a que en más de una ocasión ha habido peleas entre perros en las cuales los niños terminan interviniendo (p6, 43:43).

Es importante tomar en cuenta que en las acciones ejercidas por los docentes y personal administrativo no se ha tomado en cuenta el rol que las mascotas tienen en la vida de sus dueños, lo que ha generado, en consecuencia, poco involucramiento en las mismas. 


\section{Discusión}

La educación es un fenómeno social que está condicionado por diversos factores y estructuras como la economía, la política, el sistema educativo, las instituciones, las relaciones docente-alumno, la didáctica (Ravelo, 2013), así como el ambiente de crianza (Ardila, 2012) o contexto en el que los niños desarrollan sus habilidades; en el caso de la escuela evaluada, las condiciones socioeconómicas y culturales de la comunidad escolar representan limitaciones para el desarrollo integral de los niños.

Uno de los principales factores limitantes del contexto de esta comunidad escolar es el nivel socioeconómico, el cual influye en el ambiente de crianza, en las expectativas que los padres tienen de sus hijos, lo cual ha sido determinante para el rendimiento de estos últimos en la escuela (Formichella, 2017), pues los padres consideran a la institución principalmente como un lugar en el que pueden dejar a los niños mientras ellos trabajan; no como un espacio de crecimiento y desarrollo, y por ello se ha generado muy poco involucramiento en el cumplimiento de tareas y poco interés en el aprovechamiento de los conocimientos adquiridos en clases, pues no conciben una utilidad real de los mismos en su vida diaria, mucho menos a futuro.

Las condiciones sociales de precariedad también influyen en las expectativas que los docentes tienen acerca de las capacidades académicas de los estudiantes (Del Río, 2010), lo que impide la implementación de prácticas docentes inclusivas orientadas a eliminar las barreras de desarrollo y a formar expectativas de cambio en las condiciones de vida que su entorno les ofrece.

Ahora bien, la escuela no puede ser considerada como una institución que promueve estilos de vida saludables, pues los esfuerzos que realizan al respecto son aislados y, generalmente, únicamente dirigidos a mejorar la higiene y alimentación de los niños desde un enfoque informativo. Sin embargo, de acuerdo con el Modelo de Estilo de Vida Promotor de Salud de Nola Pender (citado en Laguado y Gómez, 2014) el estilo de vida saludable no solo implica la elección y consumo de alimentos siguiendo la pirámide alimenticia, sino que también debe atender aspectos como la realización de actividades físicas, generación de responsabilidad para estar al pendiente de la propia salud, relaciones interpersonales saludables, manejo del estrés y actividades orientadas a la autorrealización.

Resulta significativo tomar en cuenta que las mascotas tienen una influencia positiva en la salud y bienestar de las propietarios, a tal grado de que tienen un impacto en los aspectos 
Revista Iberoamericana

de las Ciencias Sociales y

Humanísticas

ISSN: $2395-7972$

psicológico, fisiológico, terapéutico y psicosocial (Wood, 2005); en lugar de tratar de sacar a los animales de la escuela, podría diseñarse una estrategia de cuidado, educación y limpieza de las mascotas, así como formas adecuadas de convivir con los animales de la comunidad y eso podría utilizarse como un currículo transversal en la formación de los alumnos.

Otro de los motivos por los cuales los intentos de los docentes por modificar algunas de las condiciones de la escuela han sido hasta cierto punto infructuosos es que no toman en cuenta el contexto que se ha descrito a lo largo de este trabajo. Ejemplo de ello son las medidas ejercidas para sacar a los perros de la escuela, sin tomar en cuenta precisamente que las mascotas tienen una influencia positiva en la salud y bienestar de los propietarios; un impacto positivo en aspectos de tipo psicológico y psicosocial, como ya se mencionó líneas arriba (Wood, 2005).

Es importante dar atención a la necesidad de mejorar las condiciones de salud, higiene y hábitos de estilo de vida saludable de los alumnos en este centro escolar, pues solo cuando las necesidades fisiológicas se encuentran debidamente satisfechas puede surgir una nueva serie de necesidades orientadas a la seguridad y autorrealización (Maslow, 1991). Sin duda estas acercan a los individuos al bienestar a través del desarrollo de su potencial humano.

Los alumnos de la escuela objeto de estudio se enfrentan a condiciones socioculturales que los ponen en situación de exclusión y vulnerabilidad. Por este motivo resulta necesario que se adopte un modelo inclusivo basado en principios para la mejora de la educación y la sociedad (Booth y Ainscow, 2015). De tal forma que se requiere promover acciones para que, además de ser una comunidad de aprendizaje, la escuela también sea una comunidad de promoción social, y así proporcionar a los alumnos herramientas y recursos personales para superar las barreras que limitan el desarrollo integral. 


\section{Revista Iberoamericana}

de las Ciencias Sociales y Humanísticas

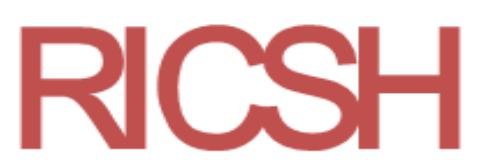

ISSN: 2395 - 7972

\section{Conclusiones}

La escuela es para una inmensidad de alumnos su única oportunidad de promoción social, entendiendo por ello el cambio de los hábitos, la cultura, los conocimientos y en sí la ideología que el contexto social impone a los integrantes de su comunidad.

Pertenecer a un contexto sociocultural da fortalezas, pero a la vez limita. No importa que se trate de un alto o muy bajo nivel, la condicionante que este impone tiene esas dos características: de potenciar en algunos aspectos y la de limitar en otros. Es como el equipamiento biológico del que disponemos para el desarrollo de la cognición humana, a la vez que nos dota para poder relacionarnos con el medio físico, nos limita para protegernos; por ejemplo, podemos escuchar solamente el espectro audible para el humano (de 20 a 20 $000 \mathrm{~Hz}$ ), más arriba o más abajo sería molesto al oído. Así, el "barrio" condiciona: favorece y limita a la vez.

En ese sentido, la escuela ubicada en un barrio con las características descritas debe representar para sus alumnos una oportunidad para romper sus limitaciones. Los alumnos no necesariamente están destinados a ser obreros (que nada de malo tendría), pero podrían estar aspirando a tener los mejores promedios, a constituirse en lectores, a tener una vida saludable, en fin, a ser mejores de lo que ahora son. Eso es trascender socialmente, y ese debería ser el papel que podrían estar ocupando los afanes cotidianos de la escuela.

Los estilos de vida saludable deben ser fomentados con persistencia, corresponsabilidad y permanencia, de lo contrario se convierte en un trabajo infructuoso. La escuela podría tener políticas y principios inclusivos que den fortaleza a un estilo de vida saludable, que trastoque la cultura impuesta por la comunidad. Esa es una función de la escuela escrita en el modelo educativo. Pero no ocurre de esa forma, lo que ocurre es lo que se presenta en los resultados, intentos de algunos de los docentes, pero con pocos resultados, porque son esfuerzos individuales, que se dan mientras ese docente no se canse de intentarlo él solo.

Con base en todo lo anterior, se ha propuesto a la institución educativa un plan de mejora que tiene como objetivo establecer alianzas en tres áreas generales: 1) promoción de la salud, 2) aprendizaje colaborativo y 3) promoción de valores inclusivos. Estas áreas constituyen las mayores oportunidades de mejora para este centro educativo, y para ello se les sugirió el diseño e implementación de acciones específicas dirigidas a lograr la 


\section{Revista Iberoamericana}

de las Ciencias Sociales y Humanísticas

ISSN: 2395 - 7972

participación de todos los miembros de esta institución, como lo son autoridades, docentes, padres de familia y estudiantes.

La escuela ha aceptado poner en marcha el plan de mejora que se diseñó para dar atención a sus necesidades; una de las principales líneas de acción es la formación de clubes colaborativos temáticos en los que se trabajan temas de estilo de vida saludable y se promueve la inclusión. Los clubes conformados son los puestos a continuación:

Club de atención a la mascota: Dirigido a todos los alumnos de la escuela. Tiene la finalidad de fomentar el respeto y cuidado de los animales, especialmente los perros que a diario acuden a la escuela durante la hora de recreo, así como promover la vida saludable al ser responsable de otros seres vivos.

Club "Lectura nutritiva": Dirigido a toda la población estudiantil. Tiene la finalidad de impulsar a los niños el amor por la lectura, así como fomentar hábitos de alimentación saludable a través de la preparación de snacks saludables por parte de las madres de familia para ofrecer durante las sesiones del club.

- $\quad$ Periódico escolar: Dirigido a los alumnos de quinto y sexto grado. Tiene como finalidad despertar en los niños el interés por la investigación y la mejora de la lectoescritura mediante la redacción de noticias y reportajes que son publicados en un documento de edición mensual que se distribuye en el pueblo y en otras escuelas de la zona escolar a la que pertenecen.

Resulta de importancia aclarar que, al momento de la realización de este artículo, el proyecto de intervención educativa continúa en fase de implementación, por lo que no se pueden presentar los resultados obtenidos. Sin embargo, se menciona dicha intervención para destacar el hecho de que el diagnóstico aquí presentado ha constituido la base para el proceso de mejora al interior de la institución. 
Revista Iberoamericana

de las Ciencias Sociales y

Humanísticas

ISSN: 2395 - 7972

\section{Referencias}

Ardila, R. (2012). ¿Habrá algún día una sociedad sin clases? La compleja relación entre habilidad cognitiva general y nivel socioeconómico. Universitas Psychologica, 11(2), 663-667. Recuperado de https://doi.org/10.11144/Javeriana.upsy11-2.hdsc.

Arrivillaga, M., Salazar, I. y Correa, D. (2003). Creencias sobre la salud y su relación con las prácticas de riesgo o de protección en jóvenes universitarios. Revista Colombiana Médica, 34(4), 186-195. Recuperado de https://www.redalyc.org/pdf/283/28334403.pdf.

Arvide, U., Rodríguez, A., Henao, S., Gómez, F. y Aguilar, C. (2013). Resultados de un programa comunitario de intervención en el estilo de vida en niños. Salud Pública de México, 55(3), S406-S414. Recuperado de https://www.redalyc.org/articulo.oa?id=10628942007.

Banz, C. (2004). La intervención psicoeducativa en la escuela y el rol de psicólogo educacional: aspectos teóricos y prácticos. Chile: Ed. Universidad Diego Portales.

Battistich, V., Solomon, D., Doing-il, K., Watson, M. and Schaps, E. (1995). Schools as Communities, Poverty Levels of Student Populations, and Students' Attitudes, Motives, and Performance: A Multilevel Analysis. American Educational Research Journal, 32(3), 627-658. $\quad$ Retrieved from https://doi.org/10.3102/00028312032003627.

Booth, T. y Ainscow, M. (2015). Guía para la educación inclusiva. Desarrollando el aprendizaje y la participación en los centros escolares. Madrid, España: FUHEM, OIE.

Campo, L., Herazo, Y., García, F., Suárez, M., Méndez, O. y Vásquez, F. (2017). Estilos de vida saludable de niños, niñas y adolescentes. Salud Uninorte, 33(3), 419-428.

Cockerham, W. (2007). New directions in health lifestyle research. International Journal of Public Health, 52(6), 327-328. Retrieved from https://link.springer.com/content/pdf/10.1007\%2Fs00038-007-0227-0.pdf.

Del Río, F. y Balladares, J. (2010). Género y Nivel Socioeconómico de los Niños: Expectativas del Docente en Formación. Revista Psykhe, 19(2), 81-90. Retrieved from http://web.a.ebscohost.com/ehost/pdfviewer/pdfviewer?vid=5\&sid=3ff5cf149c2f-4d9f-9617-2e2b0a0360bc\%40sessionmgr4008. 
Revista Iberoamericana

de las Ciencias Sociales y

Humanísticas

ISSN: 2395 - 7972

Formichella, M. y Krüger, N. (2017). Reconociendo el carácter multifacético de la educación: los determinantes de los logros cognitivos y no cognitivos en la escuela media argentina. Revista El Trimestre Económico, 84(333), 165-191. Recuperado de http://www.scielo.org.mx/pdf/ete/v84n333/2448-718X-ete-84-333-00165.pdf.

González, M., Gómez, J. J., Valtueña, J., Ortiz, J. C. and Meléndez, A. (2008). The "healthy lifestyle guide pyramid" for children and adolescents. Nutrición Hospitalaria, 23(2), 159-168.

Retrieved

from http://scielo.isciii.es/scielo.php?script=sci_arttext\&pid=S0212-

$16112008000200013 \& \operatorname{lng}=$ es\&tlng=en.

Grimaldo, M. (2012). Estilo de vida saludable en estudiantes de posgrado de Ciencias de la Salud. Psicología y Salud, 22(1), 75-87. Recuperado de https://www.uv.mx/psicysalud/psicysalud-22-1/22-

1/Mirian\%20Pilar\%20Grimaldo\%20Muchotrigo.pdf.

Instituto Nacional de Salud Pública [INSP]. (2010). Encuesta Nacional de Salud en Escolares 2008. Cuernavaca, México: Instituto Nacional de Salud Pública. Recuperado de https://www.insp.mx/images/stories/Produccion/pdf/101202_ense.pdf.

Instituto Nacional de Salud Pública [INSP]. (2012). Encuesta Nacional de Salud y nutrición 2012: Resultados generales. Ciudad de México, México: Instituto Nacional de Salud Pública.

Instituto Nacional de Salud Pública [INSP]. (2016). Encuesta Nacional de Salud y Nutrición de medio camino 2016: Informe final de resultados. Ciudad de México, México: Instituto Nacional de Salud Pública.

Laguado, E. y Gómez, M. (2014). Estilos de vida saludable en estudiantes de enfermería en la Universidad Cooperativa de Colombia. Revista Hacia la Promoción de la Salud, 19(1), 68-83. Recuperado de https://www.redalyc.org/articulo.oa?id=309131703006.

Molina, Z. y Galaviz, L. (2017). Pediculus capitis en niños de escuelas de la zona urbana de Nuevo León, México: análisis de factores asociados. Biomédica: Revista del Instituto Nacional de Salud, 37(3), 333-340. Recuperado de https://www.redalyc.org/articulo.oa?id=84354827007. 
Revista Iberoamericana

de las Ciencias Sociales y Humanísticas

ISSN: $2395-7972$

Muñoz, M. y Uribe, A. (2013). Estilos de vida en estudiantes universitarios. Psicogente, 16(30), 356-367. Recuperado de http://revistas.unisimon.edu.co/index.php/psicogente/article/view/1923.

Ravelo, E. (2013). Descripción de factores sociodemográficos y socioafectivos y su relación con el desempeño académico de los estudiantes de cuarto semestre de Psicología de una institución de educación superior. Psicogente, 16(29), 13-31. Recuperado de http://portal.unisimonbolivar.edu.co:82/rdigital/psicogente/index.php/psicogente.

Sanabria, P., González, L. A. y Urrego, D. (2007). Estilos de vida saludable en profesionales de la salud colombianos: estudio exploratorio. Revista Médica, 15(2), 207-217. Recuperado de http://www.scielo.org.co/scielo.php?pid=S0121$52562007000200008 \&$ script=sci_abstract\&tlng=es.

Secretaría de Educación Pública [SEP]. (2010). Acuerdo Nacional para la Salud Alimentaria: Estrategia contra el sobrepeso y obesidad. Programa de acción en el contexto escolar. Ciudad de México, México: Secretaría de Educación Pública.

Secretaría de Educación Pública [SEP]. (2017). Modelo Educativo para la Educación Obligatoria. Ciudad de México, México: Secretaría de Educación Pública.

Wood, L., Giles-Corti, B. and Bulsara, M. (2005). The pet connection: pets as a conduit for social capital. Social Science \& Medicine, 61(6), 1159-1173. Retrieved from https://doi.org/10.1016/j.socscimed.2005.01.017. 


\section{Revista Iberoamericana de las Ciencias Sociales y Humanísticas}

\begin{tabular}{|c|c|}
\hline Rol de Contribución & Autor (es) \\
\hline Conceptualización & $\begin{array}{l}\text { Denisse Alejandra Unzueta Vargas (Principal) Jaime } \\
\text { Fernández Escárzaga (Igual) }\end{array}$ \\
\hline Metodología & $\begin{array}{l}\text { Denisse Alejandra Unzueta Vargas (Igual) Jaime Fernández } \\
\text { Escárzaga (Principal) }\end{array}$ \\
\hline Software & NO APLICA \\
\hline Validación & $\begin{array}{l}\text { Denisse Alejandra Unzueta Vargas (Principal) Patricia Lorena } \\
\text { Martínez Martínez (Apoya) }\end{array}$ \\
\hline Análisis Formal & $\begin{array}{l}\text { Denisse Alejandra Unzueta Vargas (Principal) Jaime } \\
\text { Fernández Escárzaga (Igual) }\end{array}$ \\
\hline Investigación & $\begin{array}{l}\text { Denisse Alejandra Unzueta Vargas (Principal) Jaime } \\
\text { Fernández Escárzaga (Apoya) }\end{array}$ \\
\hline Recursos & $\begin{array}{l}\text { Denisse Alejandra Unzueta Vargas (Principal) Jaime } \\
\text { Fernández Escárzaga (Igual) }\end{array}$ \\
\hline Curación de datos & $\begin{array}{l}\text { Denisse Alejandra Unzueta Vargas (Principal) Jaime } \\
\text { Fernández Escárzaga (Igual) }\end{array}$ \\
\hline $\begin{array}{l}\text { Escritura - Preparación del } \\
\text { borrador original }\end{array}$ & $\begin{array}{l}\text { Denisse Alejandra Unzueta Vargas (principal) Jaime } \\
\text { Fernández Escárzaga (igual) Patricia Lorena Martínez } \\
\text { Martínez (igual) }\end{array}$ \\
\hline $\begin{array}{l}\text { Escritura - Revisión y } \\
\text { edición }\end{array}$ & $\begin{array}{l}\text { Denisse Alejandra Unzueta Vargas(Principal) Patricia Lorena } \\
\text { Martínez Martínez (Igual) }\end{array}$ \\
\hline Visualización & $\begin{array}{l}\text { Denisse Alejandra Unzueta Vargas (principal) Jaime } \\
\text { Fernández Escárzaga (igual) }\end{array}$ \\
\hline Supervisión & $\begin{array}{l}\text { Jaime Fernández Escárzaga (principal) Patricia Lorena } \\
\text { Martínez Martínez (apoya) }\end{array}$ \\
\hline $\begin{array}{l}\text { Administración de } \\
\text { Proyectos }\end{array}$ & NO APLICA \\
\hline Adquisición de fondos & $\begin{array}{l}\text { Denisse Alejandra Unzueta Vargas (principal) Jaime } \\
\text { Fernández Escárzaga (igual) }\end{array}$ \\
\hline
\end{tabular}

